\title{
Effect of Process Conditions on the Aerobic Biodegradation of Phenol and Paracetamol by Open Mixed Microbial Cultures
}

\author{
Davide Dionisi, "Chinedu Casmir Etteh, Materials and Chemical Engineering Group \\ School of Engineering, University of Aberdeen, Aberdeen, AB24 3UE, UK
}

\begin{abstract}
In the context of xenobiotics' removal in biological wastewater treatment processes, this study investigated the biodegradation of phenol and paracetamol as sole carbon sources by open mixed cultures. Biodegradation of these chemicals was investigated in batch and SBR (Sequencing Batch Reactor) lab-scale experiments. In batch experiments, phenol was biodegraded by unacclimated microorganisms at concentrations $0.2-0.5 \mathrm{~g} / \mathrm{l}$. The acclimation time increased with increasing phenol concentration and no biodegradation was observed with an initial concentration of $1 \mathrm{~g} / \mathrm{l}$. However, $1 \mathrm{~g} / \mathrm{l}$ of phenol was biodegraded after previous exposure of the microorganisms to lower concentrations of phenol or to yeast extract and peptone. Batch degradation of paracetamol $(0.5 \mathrm{~g} / \mathrm{l})$ only occurred after previous exposure of the microorganisms to yeast extract and peptone. SBR experiments were run with $1.0 \mathrm{~g} / \mathrm{L}$ feed concentration of phenol or paracetamol in a range of SRT (solids residence time, 1-45 $d$ for phenol and 1-145 $d$ for paracetamol). Both substrates were removed as sole carbon sources and COD removal generally increased as the SRT increased, and was higher than $90 \%$ for phenol and $80 \%$ for paracetamol. A higher fraction of the removed substrate was assimilated into microorganisms for paracetamol than for phenol with growth yields 0.51 and $0.20 \mathrm{~g} / \mathrm{g}$ COD, respectively. This study shows how process conditions (acclimation, substrate concentration, medium composition, SRT) affect the removal of phenol and paracetamol and gives guidance on their selection for the optimum biodegradation performance.
\end{abstract}

Keywords: xenobiotics, phenol, paracetamol, activated sludge, sequencing batch reactor (SBR).

*email: davidedionisi@abdn.ac.uk,phone: +44 (0)1224 272814 


\section{Introduction}

The term "xenobiotics" indicate those chemicals which are "foreign to the biosphere", i.e. man-made chemicals or naturally occurring biorefractory chemicals which, due to human activity, are present in waste or wastewaters at higher concentrations than their baseline levels [1]. The presence of xenobiotics (e.g. pharmaceuticals, detergents) in municipal and industrial wastewaters causes concern, due to their potential toxic effects, their biorecalcitrance and the possibility of accumulation in the environment [2]. Various chemical or physical technologies have been proposed or are in use for the removal of xenobiotics from wastewaters, e.g. adsorption, stripping, chemical oxidation [3]. However, these technologies have the limitations that they either only transfer xenobiotics from one phase to another without destroying them, or are very expensive and energy intensive. On the other hand, biological processes with open mixed cultures, widely used in wastewater treatment (e.g. the activated sludge process) have potentially important advantages over chemical-physical processes, i.e. they can destroy the xenobiotics with conversion to carbon dioxide and water and they are cheaper to operate [4]. Although many studies on the biodegradation of xenobiotics have been reported with pure cultures of xenobiotic-degrading bacteria, relatively limited experimental studies have been carried out on the aerobic biodegradation of xenobiotics with the open mixed microbial cultures typical of biological wastewater treatment processes [5]. Several studies investigated the removal of xenobiotics in full-scale biological wastewater treatment plants [6-8]. However, in these studies xenobiotics were usually present as a minor fraction of the total COD, therefore it was not clear whether the xenobiotics were biodegraded as primary metabolites (i.e. used for growth and microbial energy needs), 
as cometabolites or removed by chemical-physical processes (stripping or adsorption on the microorganisms), which are all possible removal mechanisms for xenobiotics in biological wastewater treatment processes [9]. On the other hand, it is important to evaluate the ability of open mixed cultures to biodegrade xenobiotics as primary metabolites, in order to design a robust and efficient biological process for xenobiotics removal [5]. Furthermore, various process parameters can in principle affect the biodegradation of xenobiotics in biological wastewater treatment processes, e.g. concentration, solids residence time (SRT), acclimation of the microorganisms. Although a number of studies have been published on the effect of these process parameters on xenobiotics removal [10,11], considering the large number of xenobiotic substances and of wide range of process conditions that can affect their removal, the literature evidence on this topic is still limited and there is need for further experimental study.

As model xenobiotics, this study investigated the biodegradation of phenol and paracetamol by open mixed cultures. Phenol is a widely used chemical and can be present in wastewaters from the chemical and petrochemical industry. Paracetamol is an important pharmaceutical present in municipal and industrial wastewaters. In this study phenol and paracetamol were used as only carbon and energy sources, in order to determine the ability of the mixed cultures to use these substances as primary metabolites. Our study investigated the effect of process parameters (substrate concentration, acclimation of the microorganisms, medium composition, SRT) on the biodegradation of phenol and paracetamol, with the ultimate aims of improving the understanding of their biodegradation and identifying which process conditions should be used to maximize their biodegradation. 


\section{Materials and methods}

Wastewaters, chemicals and inoculum

Two synthetic wastewaters were used in this study. One wastewater had phenol and one had paracetamol as only carbon source. Synthetic phenol and paracetamol with $\geq 98 \%$ purity were used and purchased from Sigma-Aldrich. The concentration of phenol was 0.2-1.0 $\mathrm{g} / \mathrm{l}$ and that of paracetamol was $0.5-1.0 \mathrm{~g} / \mathrm{l}$. In both cases nutrients the mineral medium (prepared with tap water) also included: $\mathrm{NH}_{4} \mathrm{Cl}(0.8 \mathrm{~g} / \mathrm{l}), \mathrm{K}_{2} \mathrm{HPO}_{4}(3.5 \mathrm{~g} / \mathrm{l})$, $\mathrm{NaH}_{2} \mathrm{PO}_{4}(2.4 \mathrm{~g} / \mathrm{l})$, thiourea $(20 \mathrm{mg} / \mathrm{l})$. The mineral medium composition was in line with the one used in our previous work [12]. In some batch experiments, as described later,

a mixture of peptone and yeast extract $(0.1 \mathrm{~g} / \mathrm{L}$ each, total $0.2 \mathrm{~g} / \mathrm{L})$ was also added. Peptone and yeast extract were of analytical grade and purchased from Fisher Scientific. The inoculum was a soil from Craibstone farm in Aberdeen, Scotland $(0.1 \mathrm{gVSS} / \mathrm{g}$ soil [12]). The soil was homogenised and sieved (150 mm size) and then stored in plastic containers at room temperature before inoculation. Microbial characterisation of this soil was reported by [13].

\section{Reactors set-up}

The reactors were glass vessels with a working volume of $0.25 \mathrm{~L}$ for the batch and $1 \mathrm{~L}$ for the SBRs and the set-up was similar as in our previous study [12]. VELP SCIENTIFIC ${ }^{\circledR}$ magnetic multi stirrer with 15 stirring positions was used to provide mixing for the reactors (magnetic stirrer) for the batch. VELP SP 311 peristaltic pumps were used to fill the reactors during fill phases and empty the reactors during effluent withdrawal phases. A Stuart CD162 magnetic stirrer and magnetic stirrer bars were used for mixing. Oxygen was supplied to the well-mixed reactors via fine bubble air diffusers from an Interpet 
Airvolution AV Air Pump. The length of the phases during the SBR cycles was controlled using a programmable 20 - 250 V Energenie Four Socket Power Management System. The runs were carried out at room temperature, the temperature in the reactors was measured and was in all cases in the range $20-22{ }^{\circ} \mathrm{C}$.

\section{Experimental procedure for batch experiments}

Batch experiments were carried out with a similar procedure as described previously [14]. The reactors were open and set up in duplicates for each substrate (phenol and paracetamol). At the start-up, $0.125 \mathrm{~g} g$ of the well-sieved dry soil was mixed with $0.250 \mathrm{~L}$ of the synthetic wastewater. The dissolved oxygen concentration was measured once a day throughout the length of the experiments. An experiment set-up made up of mineral solution and inoculum without substrate was used as a control. The experiments with yeast extract and peptone were started with the same procedure as the other batch experiments, but at the beginning only yeast extract and peptone were added $(0.1 \mathrm{~g} / \mathrm{L}$ yeast extract $+0.1 \mathrm{~g} / \mathrm{l}$ peptone). After the oxygen concentration indicated the end of the yeast extract-peptone metabolism, phenol or paracetamol were added at the desired concentrations.

\section{Experimental procedure for SBR operation}

Seven runs with phenol and six runs with paracetamol were carried out, with different values of HRT and SRT. The summary of the operating parameters of the various runs in reported in Tables 1 and 2. In Runs PhenRun 1-4 and ParRun 1-4 the SRT was set equal to the HRT. Therefore, the volume of sludge withdrawn coincided with the volume of feed fed to the reactor per day. In these runs the sludge withdrawal was done automatically using the Effluent Withdrawal pump at the end of the React phase in each cycle and no 
effluent was collected during the Effluent Withdrawal phase. In the other runs the SRT was higher than the HRT, the sludge withdrawal was done manually once per day at the end of the React phase and the appropriate volume of clarified effluent was collected by a pump at the end of the Settle phase. This procedure was the same as in our other recent paper [12]. In the runs with SRT>HRT the average SRT was calculated at the end of each run from the average steady-state concentrations of solids in the well-mixed reactor and in the effluent according to equation (1) [12]:

$$
S R T=\frac{V X}{Q_{W} X+\left(Q-Q_{W}\right) X_{E F F}}
$$

In Equation (1), $V$ is the volume of the reactor when full $(1 \mathrm{~L}), \mathrm{X}$ is the biomass concentration measured at the end of the React phase, $\mathrm{XEFF}_{\mathrm{EF}}$ is the biomass concentration in the clarified effluent collected after the Settle phase, $Q$ and $Q w$ are the feed and sludge withdrawal flow rate, respectively, on a daily basis.

The reactors were started up by mixing $5.0 \mathrm{~g}$ of the well-sieved soil with $1 \mathrm{~L}$ of wastewater feed. The cycle was initiated with the settle phase, followed by effluent withdrawal. Then the first feed was introduced and reactor operation continued according to the programmed cycle pattern. Each run was operated until the substrate and biomass concentration and the SRT had reached steady state. At the end of each run, the reactor was cleaned and a new run was started with a fresh inoculum. Sampling was done three times per week. Biomass and substrate concentration in the effluent were measured by sampling the reactors at the end of the reaction phase, while biomass concentration in the effluent was measured by sampling the collected effluents from the reactors.

Analytical methods 
Hachi HQ40d ${ }^{\circledR}$ oxygen meter and probe were used in the measurement of dissolved oxygen concentration in bioreactors throughout the experiment. Biomass concentration was measured as volatile suspended solids (VSS) as described previously [12], using a Whatman 1822 - 047 Grade GF/C glass fibre filter paper of $1.2 \mu \mathrm{m}$ pore size. Soluble COD in the effluent was measured after filtration using COD Cell Test kits (Merck).

\section{Data analysis}

The data collected from the SBR runs at steady state were used to calculate the fraction of the removed COD which was converted biomass and the coefficients $Y_{X / S}$ (growth yield) and $b$ (specific rate of endogenous metabolism), according to the procedure described in detail previously [12]. The fraction of the removed COD which was converted to biomass was calculated according to equation (2):

$$
\text { Fraction of removed COD converted to biomass }=\frac{1.42 \cdot \mathrm{HRT} \cdot \mathrm{X}}{\mathrm{SRT} \cdot\left(\mathrm{S}_{0}-\mathrm{S}\right)}
$$

The parameters $Y_{X / S}$ and $b$ were calculated according to Equation (3):

$$
\frac{S R T\left(S_{0}-S\right)}{X \cdot H R T}=\frac{1}{Y_{X / S}}+\frac{b}{Y_{X / S}} S R T
$$

In these equations $S_{0}$ and $S$ are the substrate concentration in the feed and in the effluent respectively (expressed as gCOD/L). Plots of $\frac{S R T\left(S_{0}-S\right)}{X \cdot H R T}$ vs SRT were generated for both the runs with phenol and the runs with paracetamol, obtaining $Y_{X / S}$ from the intercept and $b$ from the slope. 


\section{Results and discussion}

\section{Batch experiments}

Figure 1 shows the batch tests with phenol. Figure 1a shows the dissolved oxygen profile when the unacclimated inoculum was exposed to different phenol concentrations. In these tests, the drop in oxygen concentration indicates the start of the biodegradation of the substrate, while the point at which oxygen concentration increases indicates the removal of all the biodegradable substrate. For phenol concentrations $0.2-0.5 \mathrm{~g} / \mathrm{l}$ the dissolved oxygen showed a drop a few days after the start of the experiments, followed by an increase to values close to the initial ones. No such drop in oxygen concentration was observed when the phenol concentration was $1.0 \mathrm{~g} / \mathrm{l}$. These profiles indicate that phenol was metabolised at concentrations $0.2-0.5 \mathrm{~g} / \mathrm{l}$ but not at $1.0 \mathrm{~g} / \mathrm{l}$. From Figure $1 \mathrm{a}$ we calculated the acclimation time, defined as the time required for the microorganisms to become able to degrade phenol and measured as the time required for the oxygen concentration to drop by at least $1 \mathrm{mg} / \mathrm{l}$. This method of measuring acclimation time, although it is arbitrary, is consistent with our previous studies [14] and allows a consistent comparison between experiments done with the same methodology. Figure $1 \mathrm{~b}$ shows the acclimation time vs the initial phenol concentration. This plot indicates that the acclimation time was longer for higher phenol concentrations. By comparison, the acclimation time of the same inoculum with readily biodegradable substrates (ethanol and glucose) was $1 \mathrm{~d}$ irrespective of the substrate concentration in a range of concentrations $0.1-1.0 \mathrm{~g} / \mathrm{l}$ (data not shown). The evidence from Figures $1 \mathrm{a}$ and $1 \mathrm{~b}$ indicates that phenol inhibits its own microbial biodegradation and that the extent of inhibition increases as the phenol concentration increases, with much longer acclimation time at 0.5 than at $0.2-0.4 \mathrm{~g} / \mathrm{l}$ and 
with no acclimation at $1.0 \mathrm{~g} / \mathrm{l}$. However, Figure $1 \mathrm{c}$ shows that microorganisms become able to biodegrade $1.0 \mathrm{~g} / \mathrm{l}$ phenol after they have been previously acclimated at a lower concentration $(0.5 \mathrm{~g} / \mathrm{l})$. The same results were observed after acclimation at lower phenol concentrations ( 0.2 and $0.4 \mathrm{~g} / \mathrm{l}$, data not shown). Interestingly, microorganisms became able to degrade $1.0 \mathrm{~g} / \mathrm{l}$ of phenol after the addition of a $0.2 \mathrm{~g} / \mathrm{l}$ mixture of yeast extract and peptone (Figure 1d), even without any previous acclimation to phenol. The initial decrease in the oxygen concentration in Figure 1d was due to the biodegradation of the organic matter contained in yeast extract and peptone (which are mainly made of peptides, amino acids and carbohydrates, in addition to growth factors and vitamins). Phenol was only added and degraded after the dissolved oxygen profile increased back to the initial levels, indicating complete metabolisation of the biodegradable carbon sources in yeast extract and peptone.

Inhibition of microbial activity by phenol was observed in several other studies, however no other studies specifically investigated the effect of phenol concentration on the acclimation time, as we did in this study. Watanabe et al. [10] observed a deterioration of phenol removal in a continuous process when the phenol concentration in the feed was increased from 1 to $2 \mathrm{~g} / \mathrm{l}$, with complete breakdown of the process with phenol concentrations of $3 \mathrm{~g} / \mathrm{l}$ or higher. A similar observation was made by Kibret et al. [15]. Using batch tests (phenol concentrations in the range $0.1-3 \mathrm{~g} / \mathrm{L}$ ) on cultures acclimated with phenol, Morrat et al. [16] observed an increasing inhibition effect as the phenol concentration increased, with a strong increase in the inhibition effect when the phenol concentration was $1.0 \mathrm{~g} / \mathrm{L}$ or higher. Phenol inhibition was also observed at lower concentrations $(50 \mathrm{mg} / \mathrm{L})$ [17]. Our study shows the effect of phenol inhibition on the 
acclimation time of mixed cultures with no previous exposure to phenol and shows that inhibition can be overcome with previous exposure at lower phenol concentrations. Another interesting observation is that phenol inhibition can be overcome with previous exposure of the microorganisms to yeast extract and peptone, an effect that has not been reported previously and that can have important implications for the biological treatment of inhibitory wastewaters.

Figure 2a shows the oxygen profile when paracetamol was used as sole carbon and energy source $(0.5 \mathrm{~g} / \mathrm{l})$, indicating no acclimation of the inoculum to this substrate over the length of the experiment (over $70 \mathrm{~d}$ ). A similar profile, with no acclimation, was observed for a higher concentration of paracetamol ( $1.0 \mathrm{~g} / \mathrm{L}$, data not shown). However, microorganisms were able to degrade paracetamol after previous exposure to the yeast extract/peptone mixture (Figure $2 \mathrm{~b}$ ). The ability to biodegrade paracetamol was maintained even after multiple spikes of this substrate, without any other spikes of the yeast extract/peptone mixture after the initial one.

Although several pure culture strains have been identified which are able to biodegrade paracetamol at concentrations of up to $2.5 \mathrm{~g} / \mathrm{L}$, there is limited evidence about the ability of mixed cultures to biodegrade paracetamol, which is mostly treated by chemical physical methods when present in wastewaters [18]. Fan et al. [19] observed $92 \%$ removal of paracetamol by acclimated activated sludge in batch tests. Tambosi et al. [20] observed complete removal of paracetamol at low concentrations by an acclimated activated sludge operated in continuous mode. Similar results were observed by Sim et al. [21] (84-100 \% removal of paracetamol fed at low concentrations in wastewater treatment plants). In these studies however paracetamol was present at low 
concentrations in the influent wastewaters, which were mainly composed of biogenic substrates. Therefore these studies don't indicate whether paracetamol was used for microbial growth or for cometabolism and don't investigate the acclimation of microorganisms to this substance. Our batch results indicate the difficulty of obtaining acclimation of mixed cultures to paracetamol and the potential of yeast extract/peptone mixtures to trigger the acclimation to this pharmaceutical. These results confirm the positive effect of yeast extract/peptone mixtures in stimulating the acclimation to xenobiotics, already observed for phenol. Although no other studies have investigated the effect of yeast extract/peptone on the biodegradation of phenol or paracetamol by open mixed cultures, other studies have reported a positive effect of yeast extract addition to the mineral medium for azo dyes and p-nitrophenol $[22,23]$. It is important to note that in our study, differently than in other literature studies, phenol and paracetamol were added to the medium only after the complete degradation of the biodegradable fraction of yeast extract and peptone (Figures 1d and $2 \mathrm{~b}$ ), therefore any beneficial effect of yeast extract and peptone was not due to cometabolism but to the presence of growth factors, vitamins or to essential mineral elements contained in these substrates.

\section{SBR experiments}

Figure 3 shows the time profiles of the COD removal in the SBR runs with phenol as sole carbon source. Generally the steady state was achieved after 30-40 d of operation. Figures $3 a$ and $3 b$ refer to the runs with the shortest SRT ( 1 and $2 \mathrm{~d}$ respectively), where very little COD removal was observed. COD removal was higher (26\%), even though still low, in the run at SRT $3 \mathrm{~d}$ (Figure 3c). In order to determine whether the COD removal was limited by the residence time of the microorganisms in the reactor, after $58 \mathrm{~d}$ the 
operating mode of this run was switched to batch, with no further addition of substrate and removal of microorganisms (except for sampling). The COD removal increased significantly, showing the importance of allowing enough contact time between microorganisms and substrate. Figures $3 \mathrm{~d}-\mathrm{g}$ show the COD removal obtained in the runs with SRT in the range 4-45 d. Although in all these runs the COD removal was higher than in the runs at shorter SRT (Figures 3a-c), we can observe a certain inconsistency in COD removal, with very high COD removal in Figures 3d, 3f (SRT 4 and $34 d$, respectively) and lower removal in Figures $3 e, 3 g$ (SRT 5 and $45 d$, respectively, referring to the first part of Figure $3 \mathrm{~g}$ only, before the change in operating mode). These inconsistencies in COD removal probably indicate that other factors, in addition to the SRT, influence phenol removal in biological processes, for example the activity of the initial inoculum. Figure $3 \mathrm{~g}$ also shows the effect of operating mode on phenol removal. After the first steady state was reached (with $51 \%$ COD removal), the operating mode was switched to batch, until phenol was completely removed. This result further confirms the effect of increasing the residence time on phenol removal. When the cycle pattern was switched back to 4 cycles per day, COD removal decreased to the values obtained in the first part of the run. At day 140, the cycle pattern was switched to 1 cycle per day, obtaining a significant deterioration in COD removal. At day 210 the cycle pattern was switched to 8 cycles per day, obtaining a rapid improvement in COD removal.

Figure 4 summarises the effect of the SRT on the COD removal at steady state in the various runs with phenol (for Run Phen7 only the initial part of the run at 4 cycles per day is considered in Figure 4, for consistency with the other runs which were operated at 4 cycles per day). This figure indicates that generally phenol removal increased as the SRT 
increased, although with the already observed fluctuations at high SRT. The highest COD removal observed with phenol was $94 \%$.

Although several studies have investigated the biodegradation of phenol, only very few studies have systematically investigated the effect of SRT on phenol removal in continuous processes with open mixed cultures. Yoong et al. [24] operated an SBR with SRT in the range 4-27 d (feed phenol concentration $1.3 \mathrm{~g} / \mathrm{l}$ ), obtaining in all cases COD removal of at least $97 \%$. Nakhla et al. [25] operated fill and draw reactors fed with phenol at $0.8 \mathrm{~g} / \mathrm{L}$ in the SRT range 3-20 d, finding that a minimum SRT of $10 \mathrm{~d}$ was require to ensure stable and complete removal of phenol. Our results, obtained in the SRT range 1$45 \mathrm{~d}$, extend the range of investigated SRT, showing that SRT of at least $4 \mathrm{~d}$ is required to achieve high removal of phenol. As far as the effect of the number of cycles is concerned, to be best of our knowledge this is the first report of how the manipulation of the number of cycles can affect phenol biodegradation. The evidence that phenol degradation can be significantly improved, or made worse, by increasing or decreasing the number of cycles per day, for fixed values of the other parameters, is consistent with inhibitory effect of phenol observed in batch tests. High number of cycles correspond to low phenol concentration at the end of each feed in the SBR, whilst low number of cycles corresponds to high peak phenol concentration, with higher potential for inhibition. The number of cycles is a unique parameter of SBR, not present in continuous-flow activated sludge processes, therefore SBR technology can offer additional flexibility for the treatment of inhibitory wastewaters.

Figures 5a-f show the time profiles in the SBR runs with paracetamol as sole carbon source. The time required to reach steady state varied from $10-20 \mathrm{~d}$ to $70-80 \mathrm{~d}$. COD 
removal was very low at the shortest SRT (Run Par1, SRT $1 \mathrm{~d}$, Figure 5a), then it generally increased as the SRT increased. Figure 7 shows the effect of the SRT on the COD removal in the paracetamol runs at steady state, confirming the strong effect of this process parameter. COD removal of up to $85 \%$ was observed for paracetamol at high values of the SRT.

To the best of our knowledge, this is the first study that investigated the effect of SRT on the biodegradation of paracetamol as sole carbon source by open mixed cultures. Most published reports on paracetamol biodegradation involve pure cultures and relatively short term batch experiments [18]. Shariati et al. [26] observed complete paracetamol degradation in an airlift membrane bioreactor (feed concentration $1 \mathrm{~g} / \mathrm{L}$ ) but the SRT was not reported. Our study shows that very high COD removal can be obtained in paracetamol-fed continuous processes if the SRT is long enough. Generally a SRT of at least 3 days is required to achieve $50 \%$ COD removal and over $80 \%$ COD removal is obtained for SRT longer than 30 days. The SRT is a key design parameter in biological wastewater treatment processes, this study gives guidance on the choice of its value for paracetamol-rich wastewaters.

Figure 6 shows the fraction of the removed COD which was converted to biomass (a) (Equation (2)) and the linearised plot (b) (Equation (3)) for the calculation of the parameters $Y_{X / S}$ and $b$. For both paracetamol and phenol the fraction of the removed COD converted to biomass decreases as the SRT increases, as expected, due to the endogenous metabolism. However, this fraction is higher for paracetamol than for phenol. The calculated values of $Y_{X / S}$ and $b$ are $0.20 \mathrm{~g} / \mathrm{g} \mathrm{COD}$ and $0.025 \mathrm{~d}^{-1}$ for phenol and 0.51 $\mathrm{g} / \mathrm{gCOD}$ and $0.025 \mathrm{~d}^{-1}$ for paracetamol respectively. Since the values of $b$ are similar for 
the two substrates while the value of $Y_{X / S}$ is considerably higher for paracetamol than for phenol, the higher biomass production per unit of COD removed observed for paracetamol is mainly due to the higher growth yield and not to a different rate of endogenous metabolism. Only very limited investigation has been reported in the literature for the values of $Y_{X / S}$ and $b$ for these two substrates with open mixed cultures. Nuhoglu et al. [27] reported a value of $Y_{x / s}=0.25 \mathrm{~g} / \mathrm{gCOD}$ for phenol, in good agreement with our study. Also for phenol, Autenrieth [17] reported values of the net biomass yield in the range $0.10-0.40 \mathrm{~g} / \mathrm{gCOD}$. While it is evident that the values of the biomass yield also depend on the cultivation conditions, we can draw some observations based on the values measured in our study. The values of $Y_{X / S}$ and $b$ and the related biomass production have great importance in the design and operation of biological wastewater treatment processes. Our results indicate that plants treating high loads of paracetamol will experience higher biomass production than plants treating the same loads of phenol. On the other hand, the COD balance indicates that the fraction of the removed COD which is not converted to biomass, is oxidised by oxygen, therefore plants fed with phenol will have a larger microbial oxygen consumption than plants fed with paracetamol. The different biomass production can also have an impact on the minimum HRT which is possible to use as design value for the plant, if the minimum HRT is limited by the biomass concentration [12]. Plants fed with phenol will have a lower biomass concentration than plants fed with paracetamol, therefore, if the biomass concentration is the limiting factor in the choice of the minimum HRT, they will be able to work at a lower HRT than plants fed with paracetamol, with consequent potential savings in plant volumes and capital costs. 
An interesting observation that can be drawn from the comparison of batch and SBR experiments is that phenol and paracetamol degradation in SBR was possible with feed concentrations for which batch degradation was not observed (in the absence of previous acclimation at lower concentrations or with yeast extract and peptone). A possible explanation for this is that the inoculum concentration in the SBR experiments was larger than in the batch experiments ( $5 \mathrm{~g}$ of soil per $\mathrm{L}$ in the SBR vs $0.5 \mathrm{~g}$ of soil per $\mathrm{L}$ in batch). The higher soil concentration in the SBR experiments was due to the need to achieve a high enough concentration to guarantee good settling (settling was not required in the batch experiments). Possibly the inhibition of phenol and paracetamol is dependent on the initial concentration of microorganisms, however this deserves further investigation. 


\section{Conclusions}

Phenol and paracetamol were degraded by open mixed cultures under aerobic conditions as sole carbon and energy sources. In batch tests, the acclimation time of the microorganisms to phenol increased with increasing phenol concentrations in the range 0.2-0.5 $\mathrm{g} / \mathrm{L}$ and no acclimation was observed for higher concentrations (1.0 $\mathrm{g} / \mathrm{L})$. However, microorganisms acclimated to $1.0 \mathrm{~g} / \mathrm{L}$ phenol after previous exposure to lower concentrations or to mixtures of yeast extract and peptone. In batch tests, previous exposure to yeast extract and peptone also triggered the acclimation of the microorganisms to paracetamol, which was not otherwise degraded. In SBR experiments, both phenol and paracetamol were degraded with feed concentration of $1.0 \mathrm{~g} / \mathrm{L}$ as sole carbon sources. The degradation of phenol and paracetamol generally increased as the SRT increased, with very low biodegradation for SRT lower than $4 \mathrm{~d}$. Biomass production per unit of COD removed was significantly lower for phenol than for paracetamol, due to the lower growth yield.

Overall, this study gives further insight into our understanding of xenobiotics biodegradation in biological wastewater treatment processes and gives indications on the appropriate choice of process conditions for the biodegradation of phenol and paracetamol. 


\section{Acknowledgments}

The authors wish to acknowledge the financial support from the Petroleum Development Trust Fund (PTDF), Nigeria for this study. 


\section{References}

[1] P.K. Srivastava, P. Parihar, R. Singh, S.M. Prasad, The Risk Associated with the Xenobiotics Released Through Wastewater Reuse. In: Singh A., Prasad S., Singh R. (eds) Plant Responses to Xenobiotics. Springer, Singapore (2016).

[2] E.A. Noman, A.A.S. Al-Gheethi, B. Talip, R.M.S.R. Mohamed, H. Nagao, A.H.M. Kassim (2019). Xenobiotic organic compounds in greywater and environmental health impacts. In Management of Greywater in Developing Countries (2019) 89-108 Springer, Cham.

[3] Y. Boyjoo, V.K. Pareek, M. Ang, A review of greywater characteristics and treatment processes. Wat. Sci. Technol. 67 (2013) 1403-1424.

[4] T. Robinson, G. McMullan, R. Marchant, P. Nigam, Remediation of dyes in textile effluent: a critical review on current treatment technologies with a proposed alternative. Biores. Technol, 77 (2001) 247-255.

[5] D. Dionisi, Potential and limits of biodegradation processes for the removal of organic xenobiotics from wastewaters, ChemBioEng Rev. 1 (2014) 67-82.

[6] A. Katsoyiannis, C. Samara (2004). Persistent organic pollutants (POPs) in the sewage treatment plant of Thessaloniki, northern Greece: occurrence and removal. Wat. Res. 38 (2004) 2685-2698.

[7] L. Hernández Leal, N. Vieno, H. Temmink, G. Zeeman, C.J. Buisman, Occurrence of xenobiotics in gray water and removal in three biological treatment systems, Env. Sci. Technol. 44 (2010) 6835-6842.

[8] H. Al Qarni, P. Collier, J. O'Keeffe, J. Akunna, Investigating the removal of some pharmaceutical compounds in hospital wastewater treatment plants operating in Saudi Arabia. Env. Sci. Poll. Res. 23 (2016) 13003-13014.

[9] G. Byrns, The fate of xenobiotic organic compounds in wastewater treatment plants, Wat. Res. 35, (2001) 2523-2533.

[10] K. Watanabe, S. Hino, N. Takahashi, Responses of activated sludge to an increase in phenol loading. J. Ferm. Bioeng. 82 (1996) 522-524.

[11] M. Clara, B. Strenn, B, O. Gans, E. Martinez, N. Kreuzinger, H. Kroiss, Removal of selected pharmaceuticals, fragrances and endocrine disrupting compounds in a membrane bioreactor and conventional wastewater treatment plants. Wat. Res. 39 (2005) 4797-4807. 
[12] D. Dionisi, A.A. Rasheed, Maximisation of the organic load rate and minimisation of oxygen consumption in aerobic biological wastewater treatment processes by manipulation of the hydraulic and solids residence time, J. Wat. Proc. Eng. 22 (2018) 138-146.

[13] A.K. Bartram, X. Jiang, M.D. Lynch, A.P. Masella, G.W. Nicol, J. Dushoff, J.D. Neufeld, Exploring links between $\mathrm{pH}$ and bacterial community composition in soils from the Craibstone experimental farm, FEMS Microbiol. Ecol. 87 (2014), 403-415.

[14] C. Uzukwu, D. Dionisi, Comparison of the Biodegradation of n-alkanes and Readily Biodegradable Substrates Using Open Mixed Culture under Aerobic, Anoxic and Anaerobic Conditions, Int. J. Env. Bior. Biodegrad., 5 (2017) 65-76.

[15] M. Kibret, W. Somitsch, K.H. Robra, Characterization of a phenol degrading mixed population by enzyme assay. Wat. Res. 34 (2000) 1127-1134.

[16] B. Marrot, A. Barrios-Martinez, P. Moulin, N. Roche, Biodegradation of high phenol concentration by activated sludge in an immersed membrane bioreactor. Biochem. Eng. J. 30 (2006) 174-183.

[17] R.L. Autenrieth, J.S. Bonner, A. Akgerman, M. Okaygun, E.M. McCreary, Biodegradation of phenolic wastes, J. Haz. Mat., 28 (1991), 29-53.

[18] S. Wu, L. Zhang, J. Chen Paracetamol in the environment and its degradation by microorganisms, Appl Microbiol Biotechnol, 96 (2012) 875-884.

[19] H. Fan, J. Li, L. Zhang, L. Feng, Contribution of sludge adsorption and biodegradation to the removal of five pharmaceuticals in a submerged membrane bioreactor, Biochem. Eng. J., 88 (2014) 101-107.

[20] J. L. Tambosi, R. F. de Sena, M. Favier, W. Gebhardt, H. J. José, H. F. Schröder, R. F. P. Muniz Moreira, Removal of pharmaceutical compounds in membrane bioreactors (MBR) applying submerged membranes, Desalination, 261 (2010) 148-156.

[21] W.-J. Sim, J.-W. Lee, J.-E. Oh, Occurrence and fate of pharmaceuticals in wastewater treatment plants and rivers in Korea, Env. Poll., 158 (2010) 1938-1947.

[22] V.V. Dawkar, U.U Jadhav, G.S. Ghodake, S.P. Govindwar Effect of inducers on the decolorization and biodegradation of textile azo dye Navy blue 2GL by Bacillus sp. VUS Biodegradation. 200920 (2009) 777-787.

[23] X. Qiu, Q. Zhong, M. Li, W. Bai, B. Li, Biodegradation of p-nitrophenol by methyl parathiondegrading Ochrobactrum sp. B2, Int. Biodet. Biodegrad 59 (2007) 297-301. 
[24] E.T. Yoong, P.A. Lant, P.F. Greenfield, In situ respirometry in an SBR treating wastewater with high phenol concentrations, Wat. Res. 34 (2000), 239-245.

[25] G.F. Nakhla, I.M. Al-Harazin, S. Farooq, Critical solids residence time for phenolic wastewater treatment, Env. Technol. 15 (1994) 101-114.

[26] F. P. Shariati, M. R. Mehrnia, B. M. Salmasi, M. Heran, C. Wisniewski, M. H. Sarrafzadeh, Membrane bioreactor for treatment of pharmaceutical wastewater containing acetaminophen, Desal., 250 (2010) 798-800.

[27] A. Nuhoglu, B. Yalcin, Modelling of phenol removal in a batch reactor, Proc. Biochem., 40 (2005) 1233-1239. 
Table 1. Operating parameters for the SBRs treating the phenol wastewater.

\begin{tabular}{|c|c|c|c|c|c|c|c|}
\hline \multirow{2}{*}{ Run } & \multirow{2}{*}{$\begin{array}{l}\text { HRT } \\
\text { (d) }\end{array}$} & \multirow{2}{*}{$\begin{array}{c}\text { Sludge } \\
\text { withdrawal } \\
(\mathrm{mL} / \mathrm{d})\end{array}$} & \multirow{2}{*}{$\begin{array}{l}\text { Cycles } \\
\text { per } \\
\text { day }\end{array}$} & \multicolumn{4}{|c|}{$\begin{array}{l}\text { Length of Phases } \\
\text { (hours) }\end{array}$} \\
\hline & & & & Fill & React & Settle & $\begin{array}{c}\text { Effl } \\
\text { withdrawal }\end{array}$ \\
\hline PhenRun 1 & 1 & 1,000 & \multirow{6}{*}{4} & \multirow{5}{*}{0.033} & \multirow{6}{*}{5} & \multirow{5}{*}{0.934} & \multirow{6}{*}{0.033} \\
\hline PhenRun 2 & 2 & 500 & & & & & \\
\hline PhenRun $3^{*}$ & 3 & 333 & & & & & \\
\hline PhenRun 4 & 4 & 250 & & & & & \\
\hline PhenRun 5 & 4 & 100 & & & & & \\
\hline PhenRun 6 & 4 & 0 & & & & & \\
\hline \multirow{3}{*}{ PhenRun $7^{\star}$} & \multirow{3}{*}{4} & \multirow{3}{*}{0} & 4 & 0.033 & 5 & 0.934 & 0.033 \\
\hline & & & 1 & 0.033 & 23 & 0.934 & 0.033 \\
\hline & & & 8 & 0.033 & 2 & 0.934 & 0.033 \\
\hline
\end{tabular}

${ }^{*}$ In PhenRun 3 and PhenRun 7 part of the run was operated in batch (see Figure 3). 
Table 2. Operating parameters for the SBRs treating the paracetamol wastewater.

\begin{tabular}{|c|c|c|c|c|c|c|c|}
\hline \multirow{2}{*}{ Run } & \multirow{2}{*}{$\begin{array}{l}\text { HRT } \\
\text { (d) }\end{array}$} & \multirow{2}{*}{$\begin{array}{c}\text { Sludge } \\
\text { withdrawal } \\
(\mathrm{mL} / \mathrm{d})\end{array}$} & \multirow{2}{*}{$\begin{array}{c}\text { Cycles } \\
\text { per } \\
\text { day }\end{array}$} & \multicolumn{4}{|c|}{$\begin{array}{l}\text { Length of Phases } \\
\text { (hours) }\end{array}$} \\
\hline & & & & Fill & React & Settle & $\begin{array}{c}\text { Effl } \\
\text { withdrawal }\end{array}$ \\
\hline ParRun 1 & 1 & 1,000 & \multirow{6}{*}{4} & \multirow{6}{*}{0.033} & \multirow{6}{*}{5} & \multirow{6}{*}{0.934} & \multirow{6}{*}{0.033} \\
\hline ParRun 2 & 2 & 500 & & & & & \\
\hline ParRun $3^{*}$ & 3 & 333 & & & & & \\
\hline ParRun 4 & 4 & 250 & & & & & \\
\hline ParRun 5 & 4 & 20 & & & & & \\
\hline ParRun 6 & 4 & 0 & & & & & \\
\hline
\end{tabular}



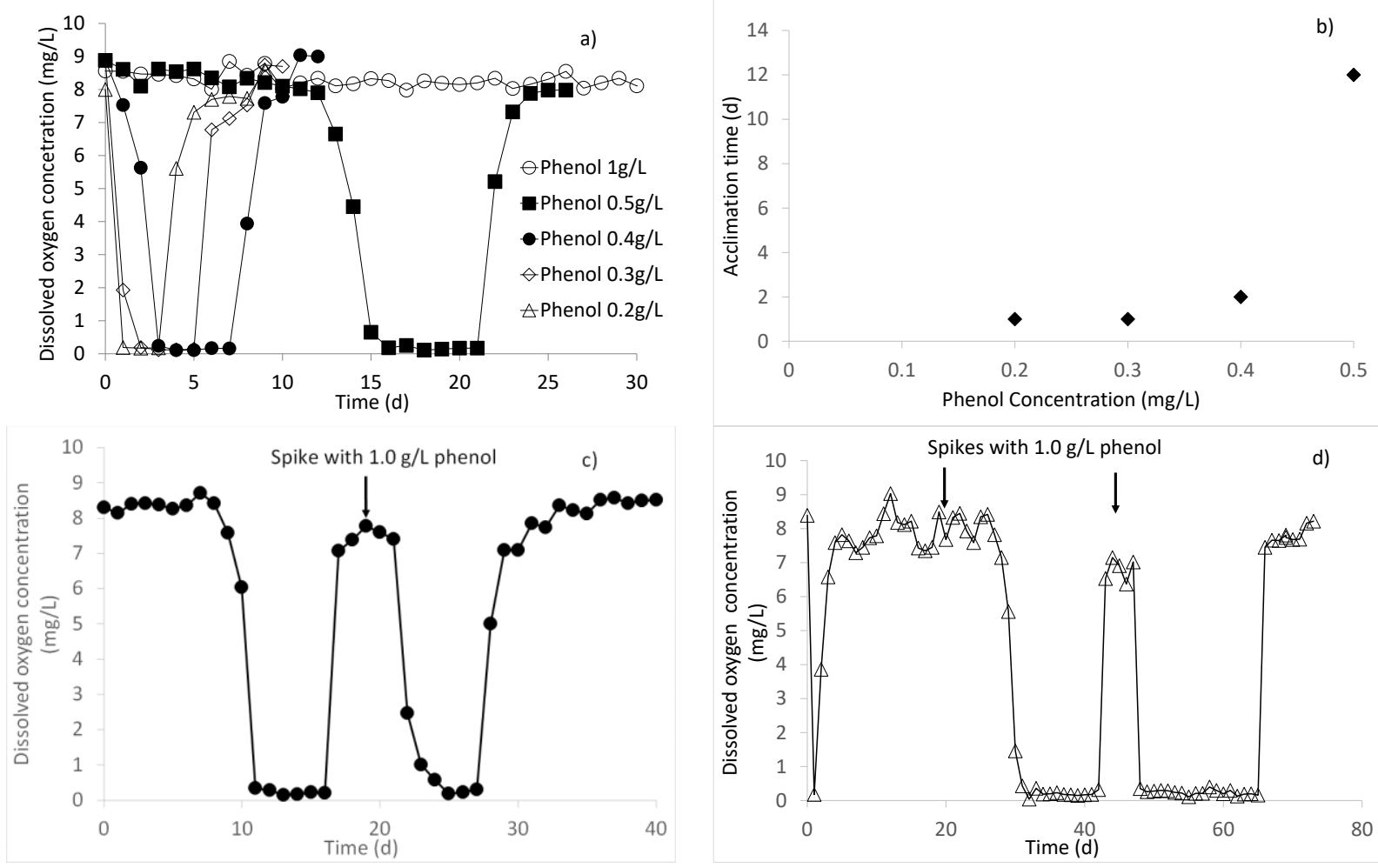

Figure 1. Batch tests with phenol. a) Dissolved oxygen profiles with different phenol concentrations (note that the experiment at $1 \mathrm{~g} / \mathrm{L}$ was carried out until day 52 with no significant change in oxygen concentration); b) Effect of initial phenol concentration on acclimation time; c) Dissolved oxygen profile with initial concentration $0.5 \mathrm{~g} / \mathrm{L}$ and then spike of $1.0 \mathrm{~g} / \mathrm{L}$; d) Dissolved oxygen profile with initial yeast extract and peptone mixture $0.2 \mathrm{~g} / \mathrm{L}$ (without phenol), followed by spikes of phenol $1.0 \mathrm{~g} / \mathrm{L}$ 

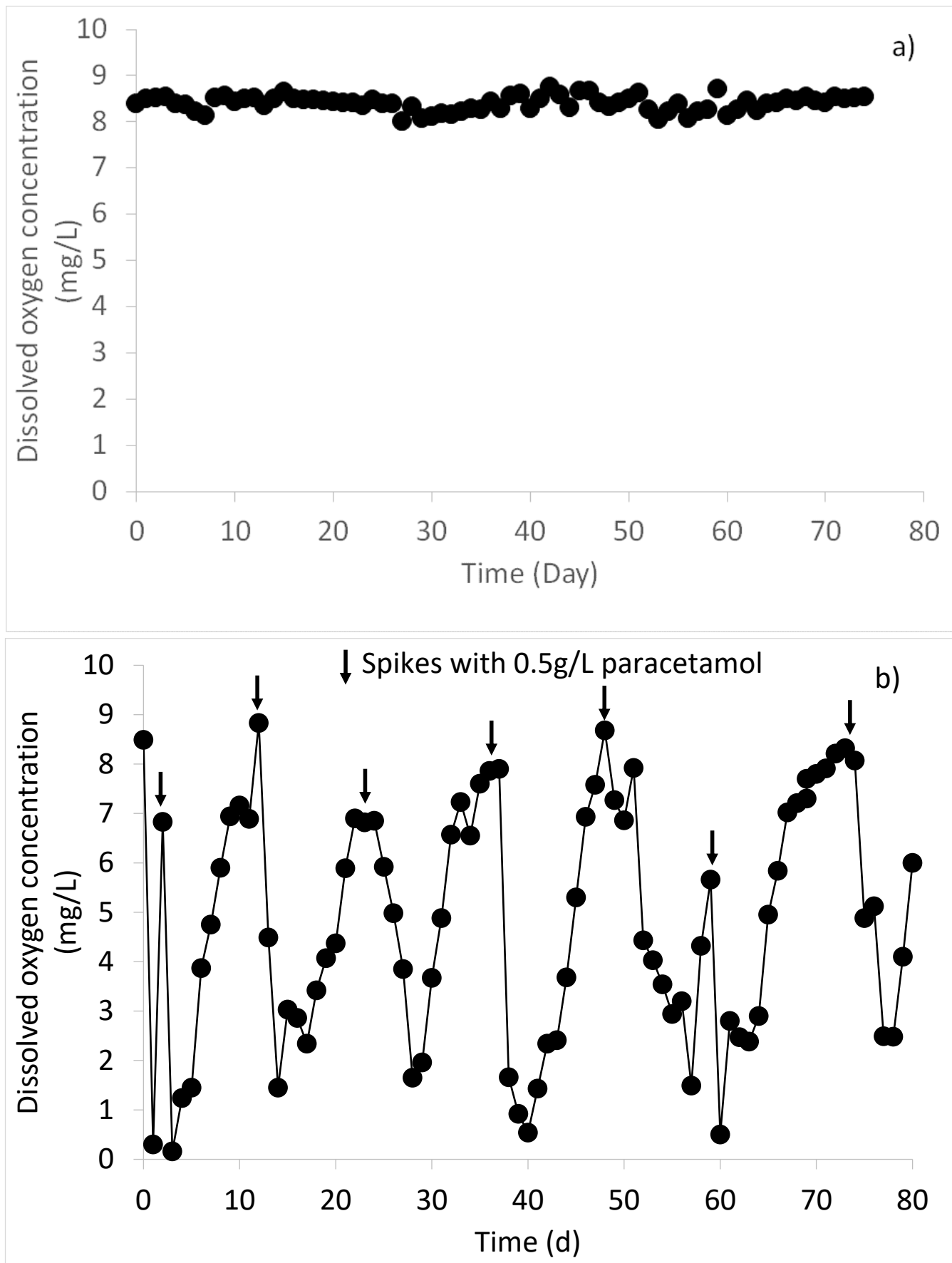

Figure 2. Batch tests with paracetamol. a) Dissolved oxygen with initial concentration $0.5 \mathrm{~g} / \mathrm{L} ; \mathrm{b}$ ) Dissolved oxygen profile with initial yeast extract and peptone mixture $0.2 \mathrm{~g} / \mathrm{L}$ (without paracetamol) followed by spikes of $0.5 \mathrm{~g} / \mathrm{L}$ paracetamol 

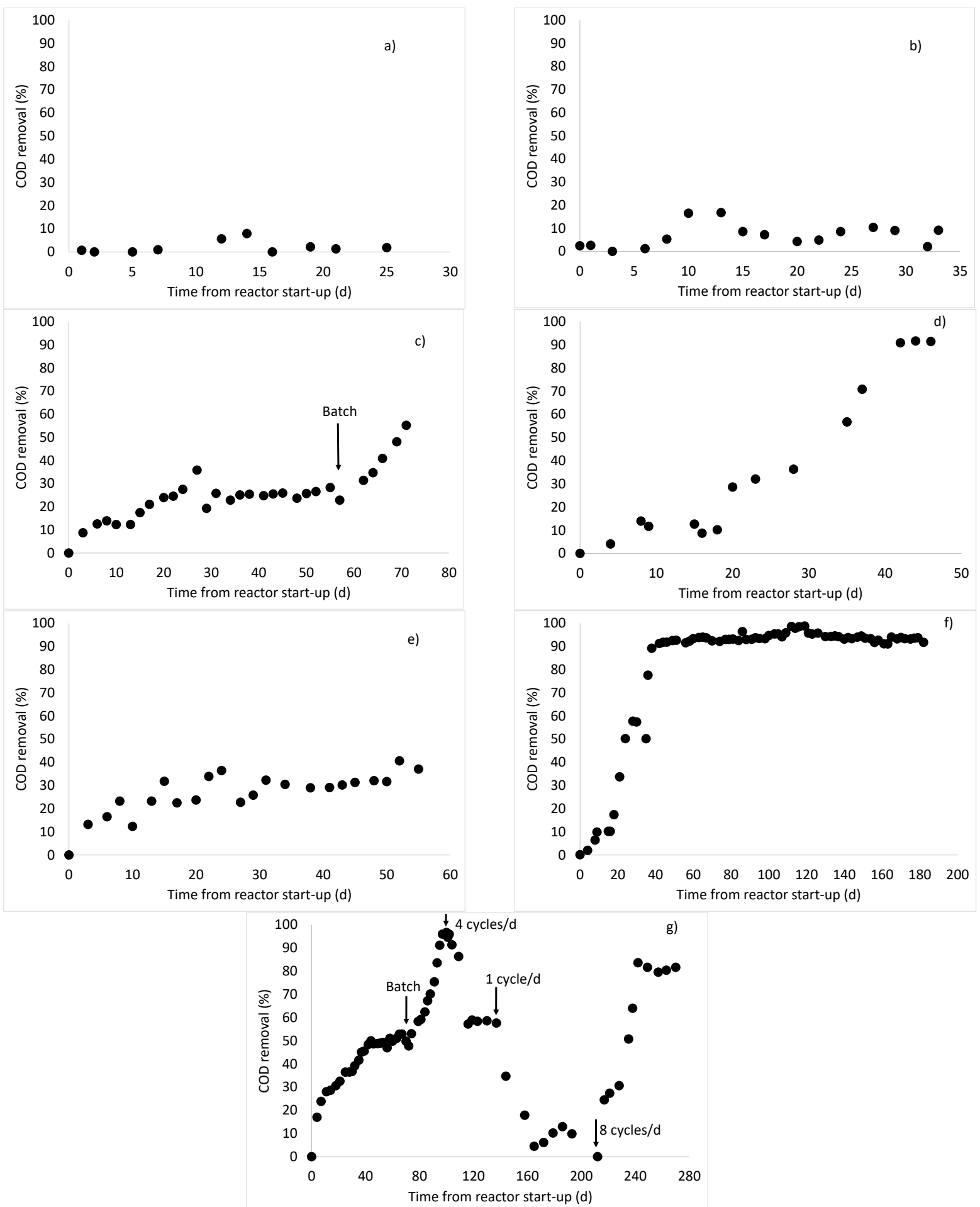

Figure 3. SBR experiments with phenol, the average SRT according to Equation (1) is in brackets. a) PhenRun $1(S R T=1 d)$; b) PhenRun $2(S R T=2 d)$; c) PhenRun 3 (SRT= $3 \mathrm{~d})$; d) PhenRun 4 $(\mathrm{SRT}=4 \mathrm{~d})$; e) PhenRun $5(\mathrm{SRT}=5 \mathrm{~d})$; f) PhenRun $6(\mathrm{SRT}=34 \mathrm{~d})$; g) PhenRun 7 (SRT= $45 \mathrm{~d})$. 


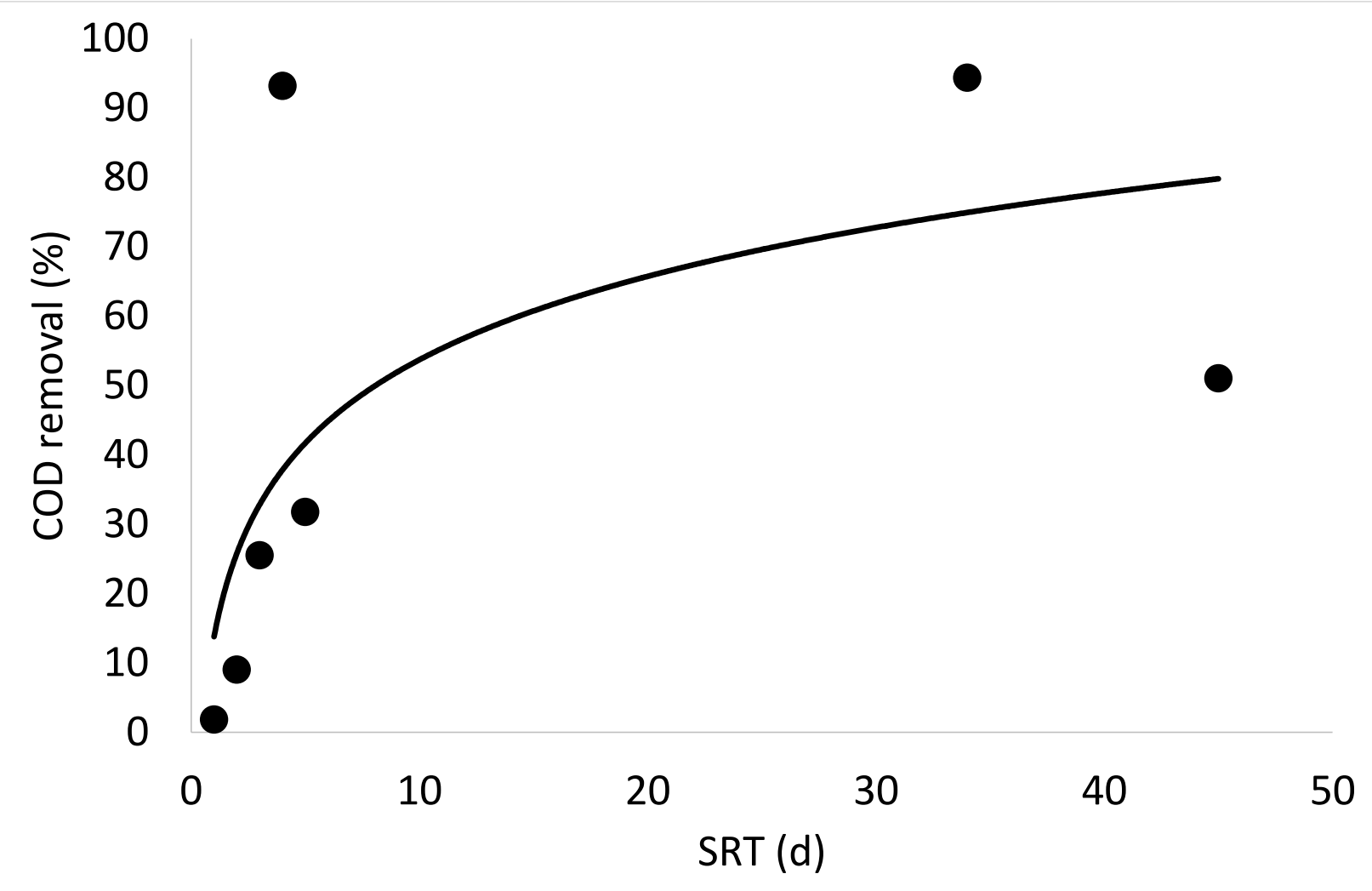

Figure 4. Effect of the SRT on COD removal in the SBR runs with phenol. 

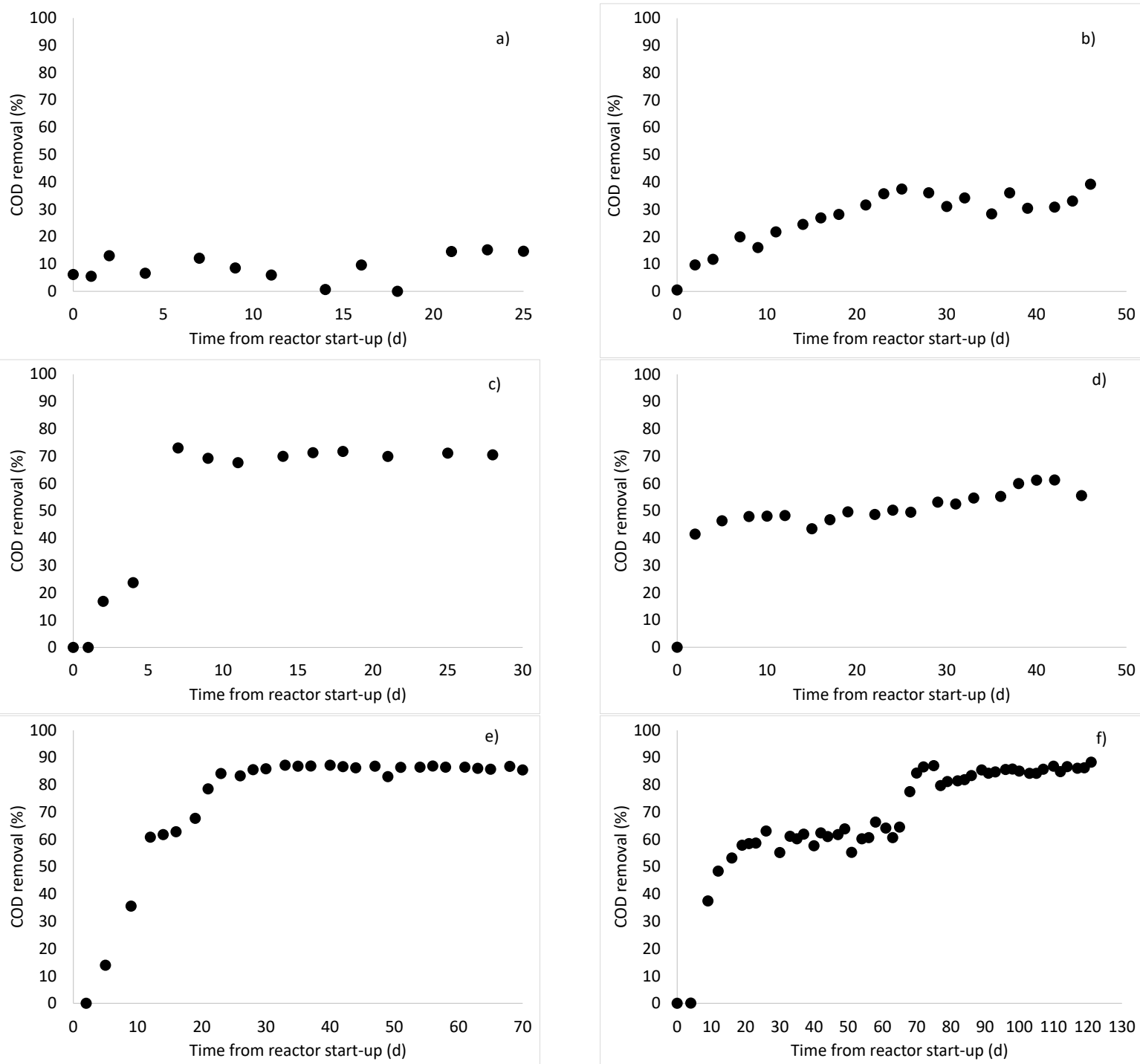

Figure 5. SBR experiments with paracetamol, the average SRT according to Equation (1) is in brackets. a) ParRun 1 (SRT = 1 d); b) ParRun $2(S R T=2 d)$; c) ParRun 3 (SRT= 3 d); d) ParRun $4(\mathrm{SRT}=4 \mathrm{~d})$; e) ParRun 5 (SRT= $39 \mathrm{~d})$; f) ParRun 6 (SRT= $145 \mathrm{~d}$ ). 


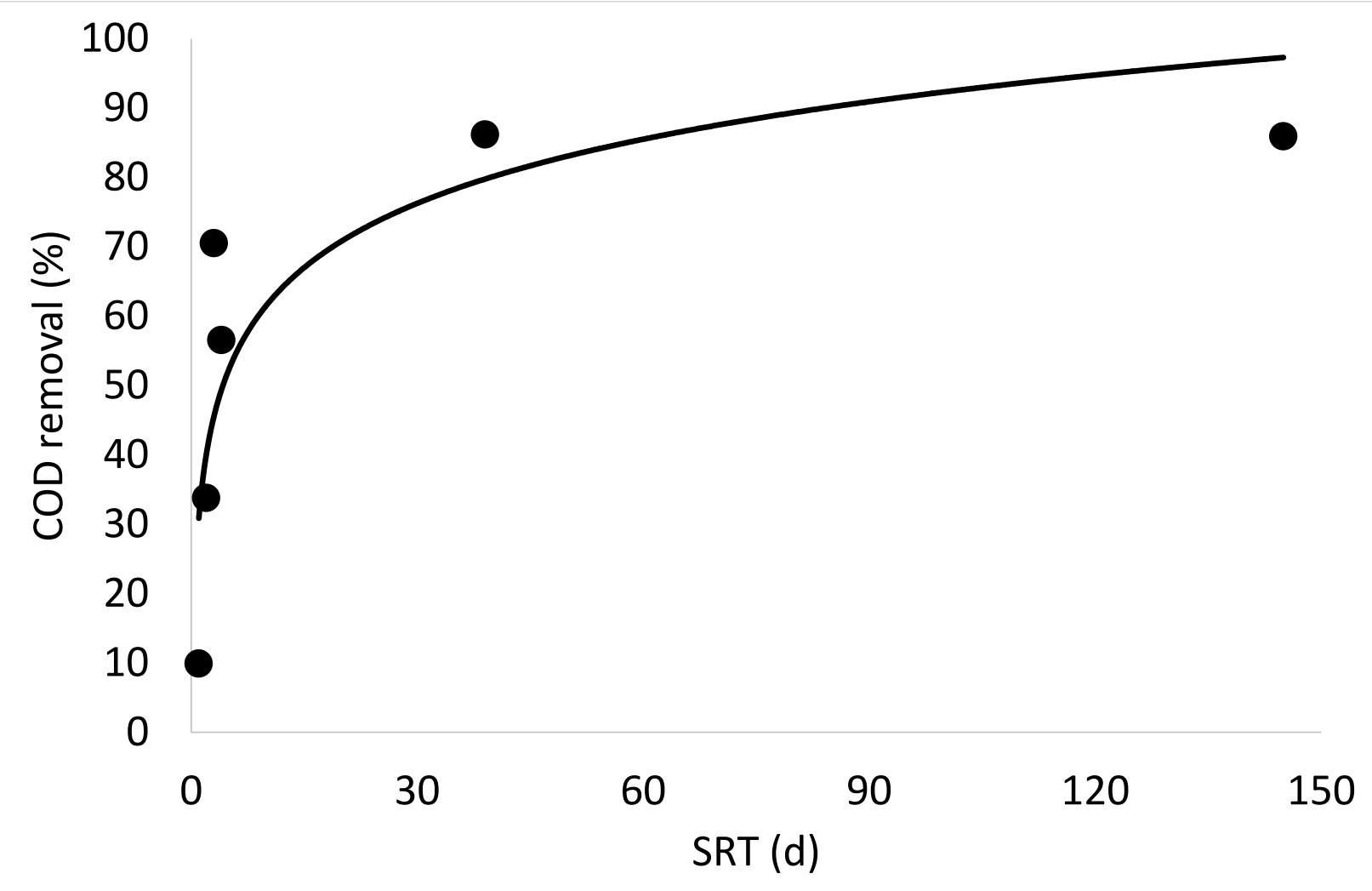

Figure 6. Effect of the SRT on COD removal in the SBR runs with paracetamol. 

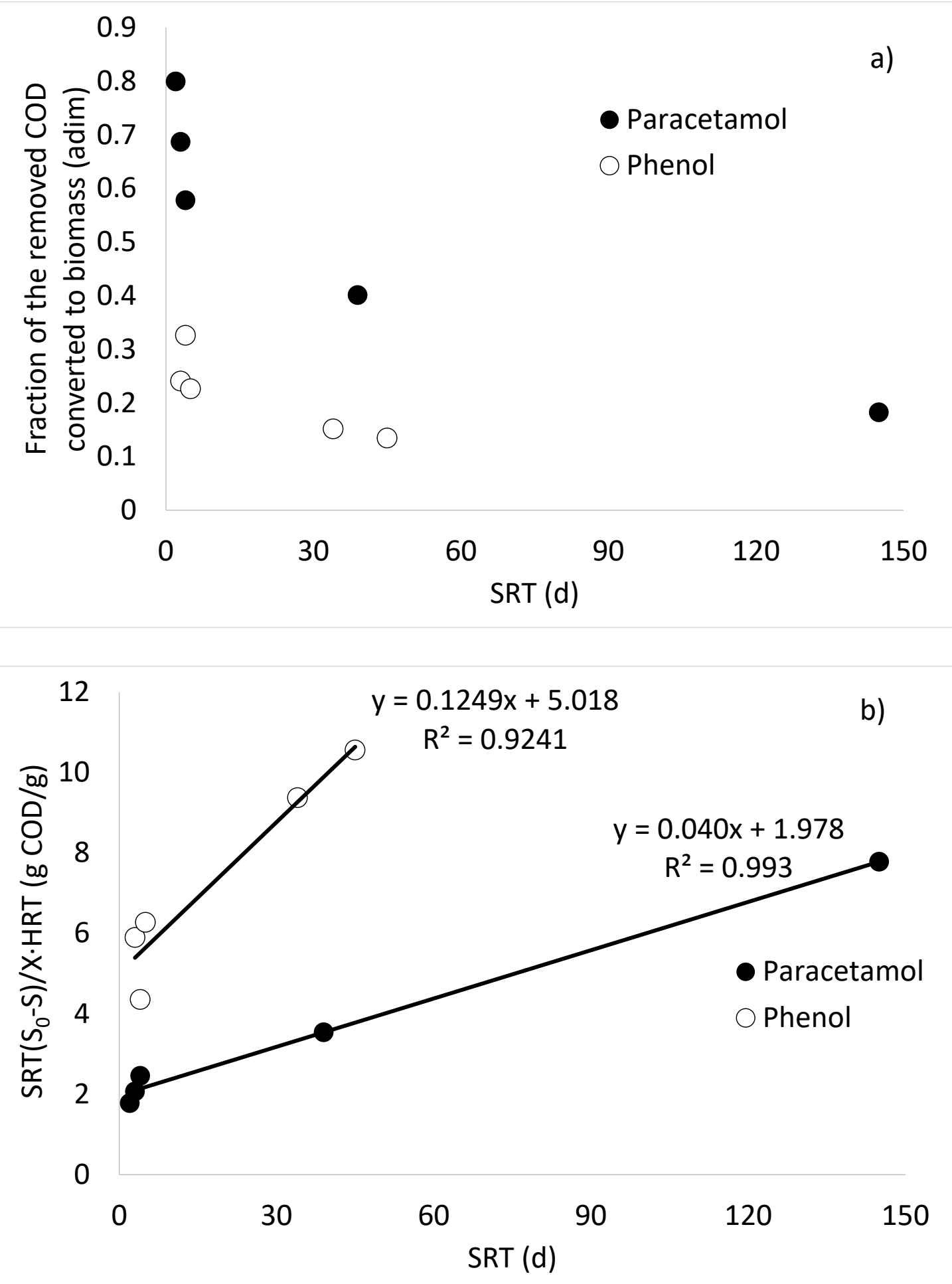

Figure 7. Analysis of the SBR data. a) Fraction of the removed COD converted to biomass; b) Linearised plot according to Equation (3). 